\title{
Investigating the Relationship between Food Pairings and Plate Waste from Elementary School Lunches
}

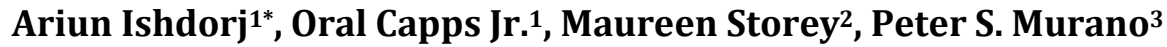 \\ ${ }^{1}$ Department of Agricultural Economics, Texas A\&M University, College Station, TX, USA \\ ${ }^{2}$ Alliance for Potato Research and Education, McLean, VA, USA \\ ${ }^{3}$ Department of Nutrition and Food Sciences, Texas A\&M University, College Station, TX, USA \\ Email: ${ }^{*}$ aishdori@tamu.edu
}

Received 29 June 2015; accepted 21 August 2015; published 26 August 2015

Copyright (C) 2015 by authors and Scientific Research Publishing Inc.

This work is licensed under the Creative Commons Attribution International License (CC BY).

http://creativecommons.org/licenses/by/4.0/

(c) (i)

Open Access

\section{Abstract}

Plate waste, defined as the quantity of edible food left uneaten after a meal, is a challenge for schools participating in the National School Lunch Program (NSLP). The new nutrition standards in the NSLP of United States Department of Agriculture (USDA) were implemented at the beginning of school year (SY) 2012-2013. School foodservice authorities were concerned that the new standards would result in increased plate waste and reduced participation, especially by students who paid full prices for lunch. There are many reasons for plate waste, including students' dislike of the foods served, the composition of meals, the environment in which students are eating, the lack of time to eat, or perhaps other factors. The objective of this study was to examine the relationship between entrée/vegetable "pairings" and plate waste by elementary school students pre- and post-implementation of the new school meal standards. Plate waste was measured to determine which entrée/vegetable pairs produced the least amount of waste. Plate waste of 144 and 305 entrée/vegetable pairings was analyzed, pre- and post-implementation, respectively. Our results indicated that more nutritious meals were offered during the post-implementation period. The new school meal standards led to no significant changes in entrée plate waste, but vegetable plate waste increased by $5.6 \%$. As such, increases in the combined entrée/vegetable plate waste were evident from $40.4 \%$ pre-implementation to $43.5 \%$ post-implementation. The top five vegetables in terms of popularity were all starchy vegetables, the majority of which were potatoes in various processed forms. The least popular vegetables were dark-green leafy vegetables, such as steamed broccoli, both pre- and post-implementation. Chicken nuggets were the most popular entrée and were wasted the least. Understanding the dynamics of food pairings and providing desirable entrée and vegetable pairings can help reduce waste from school lunches.

\footnotetext{
Corresponding author.
} 


\section{Keywords}

\section{Food Pairings, National School Lunch Program, Nutrients, Plate Waste, Vegetables}

\section{Introduction}

Most children do not consume enough fruits and vegetables in their diet [1]. Increased consumption of fruits and vegetables may reduce the risk of developing certain diet-related diseases in adulthood [2]. Children learn about healthy eating from their parents, extended family, schools, and communities in which they live. In this regard, the U.S. Department of Agriculture's (USDA) School Meal Programs-the National School Lunch Program (NSLP) and the School Breakfast Program (SBP), are in a unique position to help improve the nutritional quality of diets of school children [3]. The NSLP is the second largest food assistance program in the United States with federal spending of more than 11 billion dollars per year. It is a valuable nutrition safety net for school-age children that provides free, reduced or full-price ${ }^{1}$ lunches to more than 31 million children each school day. In 2014, more than $70 \%$ of lunches served were free or reduced-price [1] [3].

To be eligible for Federal reimbursement, all lunches served in the NSLP must meet a defined set of nutrition standards. During the 2012-2013 school year, new school meal nutrition standards were implemented in accordance with USDA regulations stemming from the Healthy, Hunger-Free Kids Act (HHFKA) of 2010 and the 2010 Dietary Guidelines for Americans [2] [4]. The new USDA guidelines for school meals were designed to improve the nutrient density of foods offered and set standards for calories, sodium, and saturated fat. In addition, among other requirements, the guidelines required serving a greater variety and larger portion sizes of fruits and vegetables [4].

School foodservice authorities were concerned that plate waste would increase as a consequence of the new regulations. Plate waste, defined as the quantity of edible food left uneaten after a meal, has been a persistent problem for schools at all grade levels [5]. Moreover, the Government Accountability Office challenged the new school meal standards as potentially contributing to greater plate waste [6].

It is not clear why students waste as much food as they do. Many factors may contribute to the waste, including a dislike or unfamiliarity with the foods served, the environment in which students are eating, lack of time to eat, or many other factors. One factor that has not been explored is the meal composition or pairings of certain foods that could enhance appeal, palatability and acceptability of the meal and lead to less plate waste. We, therefore, hypothesize that pairings of certain entrées and vegetables may reduce overall food waste.

The objective of this study was to examine the relationship between food pairings, i.e., entrées and vegetables, and plate waste by elementary school students. While a number of plate waste studies have been conducted in schools [7]-[15], none has focused on the relationship between the pairing of foods and plate waste in school lunches. To our knowledge, this paper is the first that examines pairings of entrées and vegetables associated with plate waste. This study will provide some insights on possible strategies to reduce overall plate waste from school meals.

\section{Methods}

\subsection{Participants}

Plate waste data were collected from three elementary schools (kindergarten through fifth grade) in one school district participating in the USDA's school meal program in central Texas. Plate waste collections were conducted in two phases. Phase 1 was conducted in April and May 2012, pre-implementation of the new nutrition standards, while Phase 2 was conducted in October and November 2012, post-implementation of the new nutrition standards. This timing allowed us to evaluate plate waste from school lunches pre- and post-implementation of new school meal standards. This design corresponds to a natural experiment in that the same schools were involved prior to and after the implementation of the new standards for the NSLP. In this light, this study was observational in nature without any additional interventions.

By design, the research did not involve personal identifiers regarding the participants and was approved as

${ }^{1}$ Full priced meals are also subsidized by the government. 
exempt from committee review by the Texas A\&M University Institutional Review Board.

\subsection{Data Collection Procedure}

All lunch periods for the sampled schools were scheduled by grade (kindergarten through 5). The same menu and portion sizes were served during all lunches in all three elementary schools. In all the schools, 30 minutes was allowed for lunch and recess was scheduled after the lunch. In order to meet the requirements for the new meal standard, the school district changed the recipes of the menu items and served a greater variety of vegetables; serving sizes were not changed, however. Schools used an eight-week menu cycle both pre and post-implementation.

For each data collection day, five to ten servings of each sampled entrée and vegetable were served on "test trays" by school nutrition staff. The "test trays" were used to measure pre-weights for each entrée and each vegetable for which plate waste was collected. On average, two or more options for entrées and vegetables were provided on a given day. Before each lunch period, separate trash bins were prepared for each entrée and vegetable and lined with pre-weighed trash bags. Students finished their meals and brought their trays to the research team members waiting at the designated stations. Uneaten entrées and vegetables were separated into the corresponding trash bin and the remaining contents of the tray were discarded in the cafeteria trash can for disposal. This process was repeated for each lunch period. Aggregated waste per menu item was weighed and recorded per grade at each school.

Altogether, plate waste data from 8430 students were collected—corresponding to 4145 students pre-implementation and 4285 students post-implementation of school meal standards. A total of 144 observations of entrée/vegetable pairings repeated by grade and school (27 distinct pairings) were collected pre-implementation and 305 observations of entrée/vegetable pairings repeated by grade and school (56 distinct pairings) were collected post-implementation. Analyses of the respective entrée/vegetable pairings were conducted to determine plate waste and therefore, acceptability for particular entrée/vegetable pair subsequently.

\subsection{Plate Waste Measurement and Food Pairings}

Our data collection procedures allowed for plate waste to be examined for entrée and vegetable pairings as well as for each entrée and each vegetable separately. Plate waste (in grams) for each entrée and vegetable by grade and school was divided by the number of students choosing those items.

$$
\text { Plate Waste per Student }{ }_{j k}=\frac{\text { Total Plate Waste }_{j k}}{\text { Number of Students }}
$$

The ratio provided the plate waste per student (in grams), where $j=1$ if main entrée and $j=2$ if vegetable and $k=1, \cdots, n$ is type of main entrée or type of vegetable.

Finally, the percentage of plate waste was calculated by dividing this ratio by the pre-weight (in grams) of the entrée or vegetable item, also measured in grams.

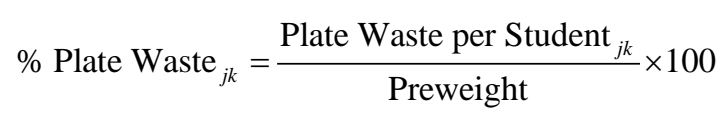

By knowing the number of participating children, plate waste was able to be expressed as a proportion or percentage.

\subsection{Statistical Analysis}

The SAS statistical analysis system (version 9.4, 2013, SAS Institute, Inc, Cary, NC) was used to create a data file with food pairings, which served as variables in conducting descriptive and statistical analysis. Mean values and standard deviations of plate waste were calculated for entrée/vegetable pairings and separately for entrées and for vegetables, for pre- and post-implementation of new school meal standards. The popularity of entrées and of vegetables was calculated as a mean number of students selecting the particular item on the data collection days. T-tests were used to determine the differences in means in popularity of entrées and vegetables, and differences in means of plate waste for entrée/vegetable pairings. The level of significance chosen was at $\alpha=$ 0.05 . 


\section{Results}

\subsection{Characteristics of Schools}

Table 1 provides characteristics of three elementary schools in our sample. These elementary schools were diverse based on race/ethnicity of the student population and percentage of students eligible to receive free/reduced-price school meals. Nearly all students in school A were eligible for free/reduced price lunches. In contrast, one-third or less of the students in school C qualified for free or reduced-price lunches. Post-implementation, a greater percentage of students in school B were eligible for free or reduced-price lunches compared to the pre-implementation period. The percentage of students eligible to receive free/reduced price meals from three elementary schools in our sample was about $66 \%$ pre-implementation and $68 \%$ post-implementation of nutrition standards. For the state of Texas, this percentage was 66\% in 2012 and for the U.S. 50\% in 2012 [16]. As reported in the School Nutrition Dietary Assessment Study-IV (SNDA-IV) report 64\% of reimbursable lunches served in elementary school during 2009-2010 school year were served for free or at reduced prices. For our sample, we found that on average $74 \%$ and $76 \%$ of reimbursable lunches served were for free or at reduced prices, pre- and post-implementation, respectively. The percent of lunches sampled on the data collection days varied from $34 \%$ to $79 \%$. Despite this variability, the data are representative of the population.

\subsection{Popularity of Entrées and Vegetables}

"Popularity" was defined as the mean number of students in our sample selecting the item on the data collection days. Popularity of the main entrées and vegetables, pre- and post-implementation of the new school meal standards, are reported in Table 2 and Table 3. On average, two or more different entrées and different vegetables selections were offered on a given day. Greater varieties of entrées and vegetables were served post-implementation of the revised standards compared with pre-implementation. The five most popular vegetables were starchy vegetables, the majority of which were potatoes in various processed forms (mashed potatoes with gravy, oven baked French fries, tater tots, and potato wedges), both pre- and post-implementation. Steamed broccoli was the least popular vegetable both pre- and post-implementation. Chicken nuggets were the most popular entrée for both periods. Burgers (plain and with cheese) were also popular pre- and post-implementation. Pre-implementation, deli sliders were the least popular entrée, whereas the sunbutter sandwich was the least popular entrée post-implementation.

\subsection{Food Pairings and Plate Waste}

We hypothesized that there was a relationship between consumption of certain entrées and vegetables that would lead to less plate waste. Our hypothesis is that if students eat less of an entrée, then they might eat more of the accompanying vegetable(s). Similarly, if students ate more of the entrée (i.e., waste less of it), then they might have eaten less of the accompanying vegetable(s).

Table 1. Total student enrollment, percent receiving free/reduced price meals, lunches served and sampled.

\begin{tabular}{|c|c|c|c|c|c|}
\hline & \multicolumn{2}{|c|}{ Total Students } & \multicolumn{2}{|c|}{ Lunches Served } & \multirow{2}{*}{$\begin{array}{c}\text { Lunches Sampled } \\
\%\end{array}$} \\
\hline & $\mathrm{N}$ & $\%$ Free/Reduced & $\mathrm{N}$ & $\%$ Free/Reduced & \\
\hline \multicolumn{6}{|c|}{ Pre-implementation of the new school meal standards } \\
\hline School A & 666 & 96 & 598 & 98 & 51 \\
\hline School B & 585 & 69 & 487 & 79 & 82 \\
\hline School C & 519 & 33 & 309 & 45 & 81 \\
\hline \multicolumn{6}{|c|}{ Post-implementation of the new school meal standards } \\
\hline School A & 677 & 99 & 609 & 99 & 36 \\
\hline School B & 601 & 74 & 437 & 84 & 65 \\
\hline School C & 516 & 31 & 302 & 45 & 61 \\
\hline
\end{tabular}


Table 2. Popularity of main entrées measured as mean number of students who selected various entrées.

\begin{tabular}{|c|c|c|}
\hline & \multicolumn{2}{|c|}{ Mean \pm Std. } \\
\hline & Pre-Implementation & Post-Implementation \\
\hline Chicken Nuggets & $60.2 \pm 9.4$ & $57.2 \pm 6.5$ \\
\hline Steak Fingers & $40.7 \pm 14.2$ & $21.3 \pm 4.3$ \\
\hline Hamburger or Cheeseburger & $40.0 \pm 18.9$ & $32.0 \pm 20.2$ \\
\hline Popcorn Chicken & --- & $37.1 \pm 10.1$ \\
\hline Pancakes and Omelets & $36.8 \pm 2.1$ & --- \\
\hline Ham and Cheese Hot Pocket & $35.9 \pm 9.9$ & --- \\
\hline Cheese or Sausage Pizza & --- & $34.2 \pm 15.9$ \\
\hline Pepperoni Hot Pocket & $33.3 \pm 7.4$ & --- \\
\hline Beef \& Bean Burrito & --- & $32.0 \pm 15.8$ \\
\hline Cheese or Stuffed Crust Pizza & $30.8 \pm 11.2$ & --- \\
\hline Hot Dog on a Bun & $25.3 \pm 7.4$ & $10.0 \pm 4.5$ \\
\hline Chicken Tender Snack Wrap & --- & $23.0 \pm 4.5$ \\
\hline Cheese or Pepperoni Pizza & --- & $20.3 \pm 11.3$ \\
\hline BBQ on a Bun & $16.9 \pm 13.4$ & $10.5 \pm 3.5$ \\
\hline Baked Chicken on Bone & --- & $16.2 \pm 6.4$ \\
\hline Grilled Chicken Sandwich & $16.0 \pm 5.8$ & $13.0 \pm 4.7$ \\
\hline Deli Wrap & --- & $16.0 \pm 4.8$ \\
\hline Hamburger & $14.3 \pm 6.8$ & --- \\
\hline Breaded Chicken Sandwich & $14.0 \pm 7.1$ & $14.6 \pm 9.3$ \\
\hline Toasted Ham and Cheese Sandwich & $13.5 \pm 3.3$ & --- \\
\hline Corn Dog on a Stick & $12.0 \pm 2.8$ & $41.8 \pm 14.6$ \\
\hline Chicken Spaghetti & --- & $10.8 \pm 3.4$ \\
\hline Munchables & $10.0 \pm 5.7$ & --- \\
\hline Deli Sandwich & --- & $8.3 \pm 3.0$ \\
\hline Chicken Fried Steak Sandwich & --- & $8.1 \pm 3.9$ \\
\hline Mini Chef Salad & --- & $6.5 \pm 2.1$ \\
\hline Mini Sub Sandwich & --- & $6.1 \pm 4.4$ \\
\hline Chef Boyardee Ravioli & $5.7 \pm 2.6$ & $7.8 \pm 4.1$ \\
\hline Deli Sliders & $4.6 \pm 1.9$ & --- \\
\hline Cheese Stuffed Bread Stick & --- & $4.6 \pm 2.1$ \\
\hline Italian Spaghetti \& Meat Sauce & --- & $4.5 \pm 3.4$ \\
\hline Cheesy Baked Potato & --- & $4.1 \pm 1.6$ \\
\hline Sunbutter Sandwich & --- & $3.3 \pm 1.9$ \\
\hline
\end{tabular}

Values are means \pm standard deviations. 
Table 3. Popularity of vegetables measured as mean number of students who selected various vegetables.

\begin{tabular}{|c|c|c|}
\hline & \multicolumn{2}{|c|}{ Mean \pm Std. } \\
\hline & Pre-Implementation & Post-Implementation \\
\hline Mashed Potatoes w gravy & $43.1 \pm 15.2$ & $29.7 \pm 21.1$ \\
\hline French Fries & $33.1 \pm 19.1$ & $24.1 \pm 12.1$ \\
\hline Tater Tots & $24.4 \pm 13.2$ & $35.4 \pm 18.0$ \\
\hline Corn on the Cob & $23.2 \pm 15.5$ & $31.3 \pm 13.4$ \\
\hline Whole Kernel Corn & --- & $18.4 \pm 15.7$ \\
\hline Sweet Potato Fries & --- & $16.3 \pm 10.4$ \\
\hline Pork \& Beans & --- & $14.4 \pm 8.8$ \\
\hline Potato Wedges & $14.4 \pm 6.0$ & $31.5 \pm 22.0$ \\
\hline Steamed Broccoli w/cheese Sauce & --- & $14.1 \pm 6.7$ \\
\hline Green Peas & $13.3 \pm 9.2$ & $4.9 \pm 3.1$ \\
\hline Green Beans & $13.3 \pm 8.0$ & $15.0 \pm 10.5$ \\
\hline Ranch Style Beans & $13.2 \pm 10.5$ & $6.5 \pm 3.8$ \\
\hline Mixed Normandy Vegetables & --- & $11.8 \pm 6.6$ \\
\hline Baked Beans & --- & $10.0 \pm 5.9$ \\
\hline Whole dill pickle & $10.0 \pm 5.7$ & $8.2 \pm 5.4$ \\
\hline Veggie Dippers & $7.1 \pm 5.1$ & $6.8 \pm 5.3$ \\
\hline Raw Baby Carrots and Celery & --- & $6.1 \pm 2.8$ \\
\hline Sonoma Vegetables & --- & $6.0 \pm 3.4$ \\
\hline Garden Salad w/ranch on the side & --- & $4.8 \pm 2.4$ \\
\hline Broccoli florets & --- & $3.8 \pm 1.6$ \\
\hline Raw Sweet Potato Sticks & --- & $3.5 \pm 1.4$ \\
\hline Steamed Broccoli & $3.7 \pm 2.2$ & $3.5 \pm 1.3$ \\
\hline
\end{tabular}

Values are means \pm standard deviations.

Figure 1 illustrates the relationship between plate waste of burgers and vegetables. As shown, burgers were a popular entrée pre- and post-implementation. Greater plate waste from burgers was observed when this popular entrée was paired with a popular vegetable like tater tots or oven-baked French fried potatoes—each having relatively low plate waste. In contrast, the burger was wasted less when paired with a less popular vegetable like sweet potato fries. The relationship between green beans and entrée plate is reported in Figure 2. Green beans were wasted the most when paired with chicken nuggets - the most popular and least wasted entrée, pre- and post-implementation. However, when green beans were paired with steak fingers—a less popular entrée-plate waste of the vegetable was reduced.

Total plate waste for all entrée and vegetable pairings in our sample pre- and post- implementation of new school meal standards, respectively, are provided in Table 4 and Table 5.

Overall, the pairing of deli sliders with corn on the cob resulted in the highest combined plate waste (62.5\%), and the pairing of corn dog on a stick with tater tots resulted in the lowest combined plate waste (21\%) (Table 4). Plate waste for entrées was the lowest for chicken nuggets (7.6\%) paired with green beans and highest for deli sliders (44.3\%) served with corn on the cob. Serving deli sliders as a main entrée and corn on the cob as a 

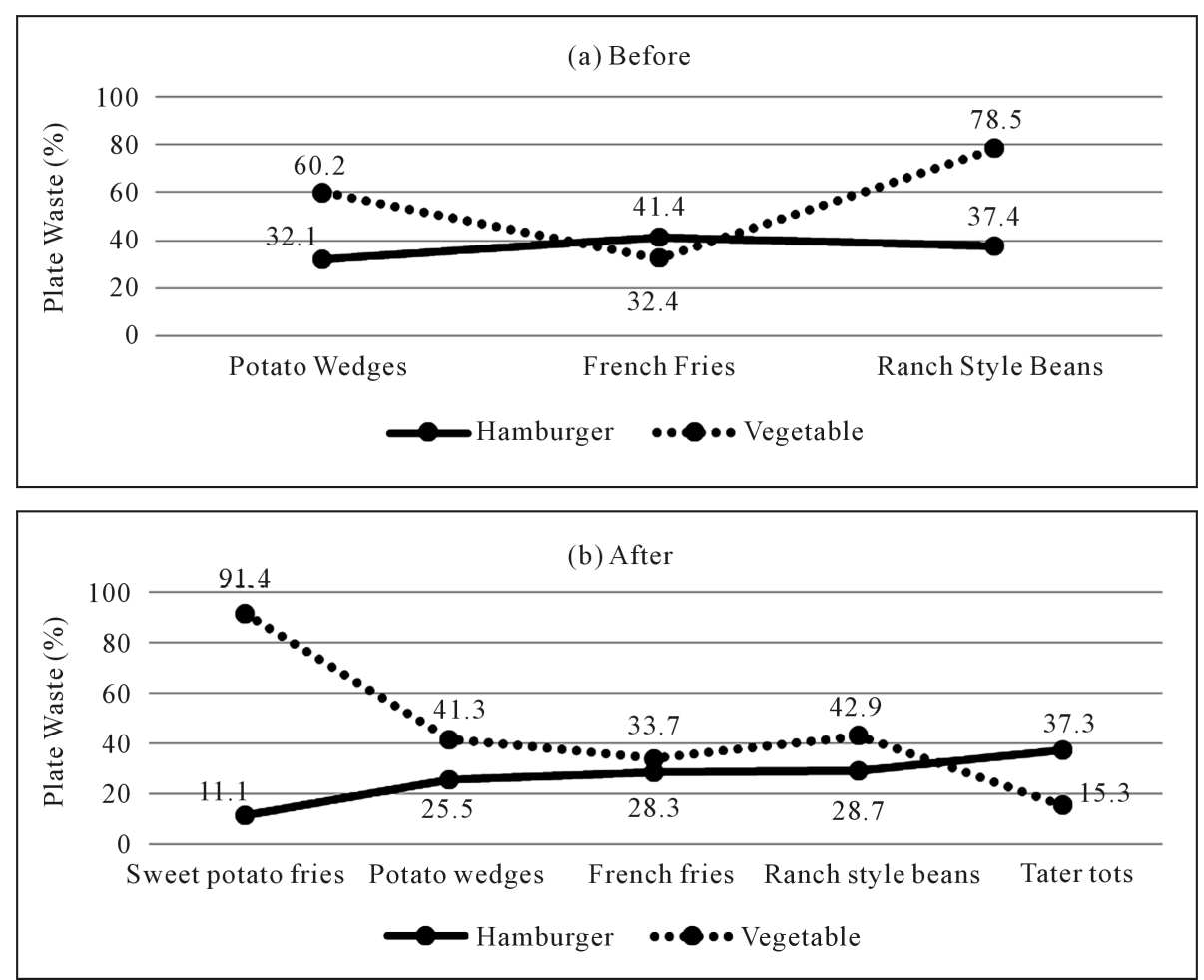

Figure 1. Relationship between hamburgers and vegetable plate waste (a) Pre-implementation of the new school meal standards; (b) Post-implementation of the new school meal standards.
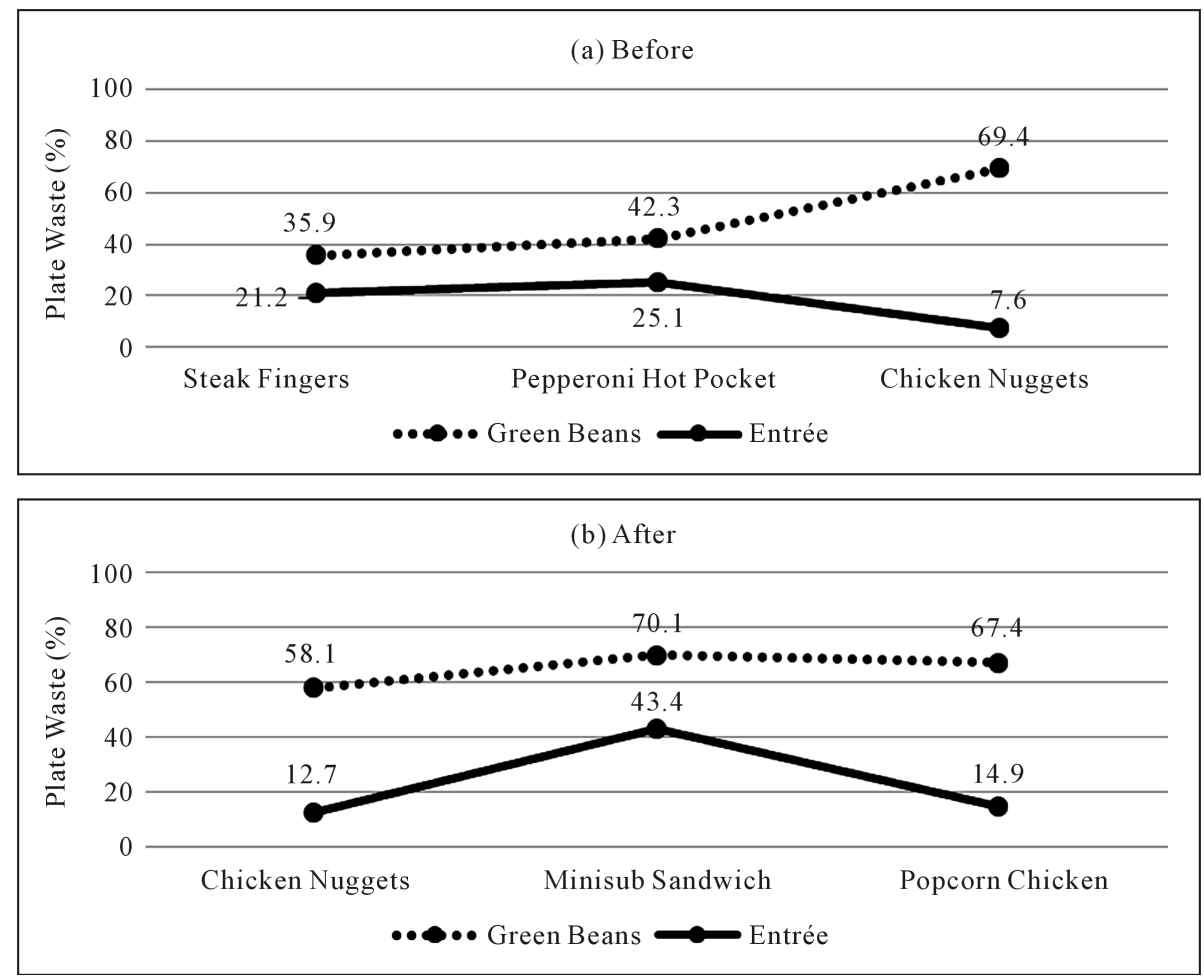

Figure 2. Relationship between green beans and entrée plate waste (a) Pre-implementation of the new school meal standards; (b) Post-implementation of the new school meal standards. 
Table 4. Vegetable and entrée pairings percent plate waste, pre-implementation of the new school meal standards.

\begin{tabular}{|c|c|c|c|c|c|}
\hline Vegetables & Main Entrées & $\mathrm{n}$ & $\begin{array}{c}\text { Vegetable } \% \\
\text { Wasted } \pm \text { Std. }\end{array}$ & $\begin{array}{c}\text { Entrée \% } \\
\text { Wasted } \pm \text { Std. }\end{array}$ & $\begin{array}{c}\text { Total \% } \\
\text { Wasted } \pm \text { Std. }\end{array}$ \\
\hline Tater Tots & Corn Dog on a Stick & 6 & $24 \pm 18.3$ & $17.9 \pm 8.8$ & $21 \pm 13.5$ \\
\hline French Fries & Hot Dog on a Bun & 6 & $38.9 \pm 18.2$ & $15.9 \pm 9.1$ & $27.4 \pm 11.2$ \\
\hline Green Beans & Steak Fingers & 6 & $35.9 \pm 17.7$ & $21.1 \pm 5.5$ & $28.5 \pm 10.8$ \\
\hline Tater Tots & Pancakes and Omelets & 6 & $30.7 \pm 11.2$ & $27.2 \pm 3.9$ & $28.9 \pm 7.5$ \\
\hline Mashed Potatoes w Gravy & Chicken Nuggets & 5 & $49 \pm 16.2$ & $9.2 \pm 5.9$ & $29.1 \pm 10.8$ \\
\hline Veggie Dippers & Chef Boyardee Ravioli & 4 & $51.9 \pm 19.8$ & $13.1 \pm 8.3$ & $32.5 \pm 13.9$ \\
\hline Green Beans & Pepperoni Hot Pocket & 6 & $42.3 \pm 19.8$ & $25.1 \pm 9.9$ & $33.7 \pm 11.1$ \\
\hline Mashed Potatoes w Gravy & Steak Fingers & 6 & $48.4 \pm 9.3$ & $21.1 \pm 5.5$ & $34.8 \pm 6.7$ \\
\hline French Fries & Hamburger or Cheeseburger & 12 & $32.4 \pm 16.5$ & $41.4 \pm 14.5$ & $36.9 \pm 14.7$ \\
\hline Green Beans & Chicken Nuggets & 4 & $69.4 \pm 14.7$ & $7.6 \pm 5.3$ & $38.5 \pm 9.1$ \\
\hline Steamed Broccoli & BBQ on a Bun & 4 & $35.8 \pm 35.6$ & $42.3 \pm 26.4$ & $39 \pm 28.0$ \\
\hline Mashed Potatoes w Gravy & Pepperoni Hot Pocket & 6 & $56.5 \pm 10$ & $25.1 \pm 9.9$ & $40.8 \pm 8.6$ \\
\hline French Fries & Toasted ham and Cheese Sandwich & 4 & $42.4 \pm 22.1$ & $42 \pm 7.2$ & $42.2 \pm 13.7$ \\
\hline Ranch Style Beans & Breaded Chicken Sandwich & 2 & $58.5 \pm 0.4$ & $26.4 \pm 5.8$ & $42.5 \pm 2.7$ \\
\hline Potato Wedges & Grilled Chicken Sandwich & 5 & $49.4 \pm 20.2$ & $36.7 \pm 15.8$ & $43.1 \pm 13.6$ \\
\hline Veggie Dippers & Deli Sliders & 5 & $44 \pm 32.8$ & $43 \pm 7$ & $43.5 \pm 15.4$ \\
\hline Whole Dill Pickle & Munchables & 5 & $61.2 \pm 27.3$ & $26.1 \pm 23$ & $43.6 \pm 19.5$ \\
\hline Steamed Broccoli & Ham and Cheese Hot Pocket & 3 & $55.9 \pm 14.1$ & $32.4 \pm 20.3$ & $44.2 \pm 10.3$ \\
\hline Veggie Dippers & Cheese or Stuffed Crust Pizza & 11 & $64.1 \pm 18.1$ & $25.7 \pm 8.3$ & $44.9 \pm 6.8$ \\
\hline Corn on the Cob & Ham and Cheese Hot Pocket & 6 & $63.2 \pm 8.5$ & $27.7 \pm 15.1$ & $45.4 \pm 10.8$ \\
\hline Potato Wedges & Hamburger & 6 & $60.2 \pm 18$ & $32.1 \pm 9.1$ & $46.2 \pm 11.0$ \\
\hline Green Peas & Chef Boyardee Ravioli & 6 & $59 \pm 26.4$ & $42.3 \pm 25.8$ & $50.6 \pm 25.9$ \\
\hline Corn on the Cob & BBQ on a Bun & 6 & $63 \pm 12.8$ & $39.1 \pm 21.8$ & $51 \pm 15.0$ \\
\hline Green Peas & Cheese or Stuffed Crust Pizza & 3 & $78.1 \pm 6.5$ & $28.8 \pm 14.3$ & $53.4 \pm 7.6$ \\
\hline Corn on the Cob & Cheese or Stuffed Crust Pizza & 5 & $81.3 \pm 6$ & $27.1 \pm 11.6$ & $54.2 \pm 7.7$ \\
\hline Ranch Style Beans & Hamburger or Cheeseburger & 3 & $78.5 \pm 6.4$ & $37.4 \pm 10.9$ & $57.9 \pm 8.6$ \\
\hline Corn on the Cob & Deli Sliders & 3 & $80.8 \pm 5.4$ & $44.3 \pm 6.6$ & $62.6 \pm 4.5$ \\
\hline All & All & 144 & $52.12 \pm 22.5$ & $28.6 \pm 15.3$ & $40.4 \pm 14.7$ \\
\hline
\end{tabular}

\footnotetext{
${ }^{\mathrm{a}}$ Values are mean $\% \pm$ standard deviation; ${ }^{\mathrm{b}}$ Ranked from smallest to largest total percent wasted; ${ }^{\mathrm{c}}$ Here $n$ in the number of observations for each
} entrée/vegetable pairing. 
Table 5. Vegetable and entrée pairings percent plate waste, post-implementation of the new school meal standards.

\begin{tabular}{|c|c|c|c|c|c|}
\hline Vegetables & Main entrées & $\mathrm{n}$ & $\begin{array}{l}\text { Vegetable } \% \\
\text { wasted } \pm \text { Std. }\end{array}$ & $\begin{array}{c}\text { Entrée \% } \\
\text { wasted } \pm \text { Std. }\end{array}$ & $\begin{array}{c}\text { Total } \% \\
\text { wasted } \pm \text { Std }\end{array}$ \\
\hline Tater Tots & Hamburger or Cheeseburger & 6 & $15.3 \pm 9.8$ & $37.3 \pm 11.3$ & $26.3 \pm 10.2$ \\
\hline Tater Tots & Beef \& Bean Burrito & 6 & $18.4 \pm 11.5$ & $36.1 \pm 27.2$ & $27.3 \pm 19.3$ \\
\hline Mashed Potatoes w Gravy & Chicken Nuggets & 6 & $44.3 \pm 6.3$ & $12.7 \pm 7.1$ & $28.5 \pm 6.4$ \\
\hline Broccoli Florets & Hot Dog on a Bun & 7 & $44.7 \pm 25.9$ & $12.6 \pm 8.9$ & $28.6 \pm 13.9$ \\
\hline French Fries & Hamburger or Cheeseburger & 6 & $33.7 \pm 15.3$ & $28.3 \pm 7.9$ & $31 \pm 9.1$ \\
\hline Potato Wedges & Hamburger or Cheeseburger & 6 & $41.3 \pm 19.7$ & $25.5 \pm 3.6$ & $33.4 \pm 9.9$ \\
\hline Garden Salad w/Ranch & Italian Spaghetti \& Meat Sauce & 4 & $38.7 \pm 25.9$ & $28.1 \pm 29$ & $33.4 \pm 26.3$ \\
\hline Mashed Potatoes w Gravy & Popcorn Chicken & 11 & $54.5 \pm 15.9$ & $13.6 \pm 7.8$ & $34 \pm 11.6$ \\
\hline Garden Salad w/Ranch & Cheese Stuffed Bread Stick & 5 & $51.3 \pm 15.9$ & $17 \pm 20.1$ & $34.1 \pm 12.5$ \\
\hline Ranch Style Beans & Breaded Chicken Sandwich & 5 & $47.4 \pm 11.2$ & $21.6 \pm 11.5$ & $34.5 \pm 3.5$ \\
\hline Garden Salad w/Ranch & Chef Boyardee Ravioli & 5 & $61.7 \pm 14.5$ & $7.6 \pm 3.3$ & $34.7 \pm 5.7$ \\
\hline Green Beans & Chicken Nuggets & 6 & $58.1 \pm 9$ & $12.7 \pm 7.1$ & $35.4 \pm 4.5$ \\
\hline Steamed Broccoli & Corn Dog on a Stick & 3 & $56.5 \pm 20.3$ & $14.6 \pm 1.3$ & $35.6 \pm 10.8$ \\
\hline Ranch Style Beans & Hamburger or Cheeseburger & 6 & $42.9 \pm 26.3$ & $28.7 \pm 18.7$ & $35.8 \pm 16.9$ \\
\hline Whole Dill Pickle & Deli Wrap & 3 & $43 \pm 6.6$ & $29.7 \pm 12.6$ & $36.3 \pm 8.3$ \\
\hline Steamed Broccoli W/Cheese & Steak Fingers & 6 & $56 \pm 18.4$ & $20.8 \pm 10.6$ & $38.4 \pm 12.7$ \\
\hline Whole Dill Pickle & Mini sub Sandwich & 9 & $43.2 \pm 26.9$ & $35.6 \pm 12$ & $39.4 \pm 14.4$ \\
\hline Raw Baby Carrots and Celery & Steak Fingers & 5 & $60.4 \pm 11.3$ & $20 \pm 11.6$ & $40.2 \pm 6.6$ \\
\hline Broccoli Florets & Sea Shaped Fish Sticks & 9 & $63.3 \pm 16.2$ & $18.6 \pm 13.1$ & $40.9 \pm 10.8$ \\
\hline Green Beans & Popcorn Chicken & 12 & $67.4 \pm 17.8$ & $14.9 \pm 8.7$ & $41.1 \pm 12.2$ \\
\hline Corn on the Cob & Corn Dog on a Stick & 6 & $71 \pm 5.7$ & $14 \pm 2.6$ & $42.5 \pm 2.1$ \\
\hline Pork \& Beans & Chicken Fried Steak Sandwich & 6 & $46.6 \pm 18.2$ & $38.4 \pm 21.1$ & $42.5 \pm 18.0$ \\
\hline Veggie Dippers & Cheese or Pepperoni Pizza & 18 & $58.4 \pm 14.2$ & $26.7 \pm 8$ & $42.5 \pm 8.3$ \\
\hline Whole Kernel Corn & Mini Chef Salad & 5 & $67.2 \pm 33.4$ & $18 \pm 24.1$ & $42.6 \pm 5.9$ \\
\hline Garden Salad w/Ranch & Chicken Spaghetti & 3 & $52.4 \pm 19.5$ & $32.8 \pm 10.2$ & $42.6 \pm 14.8$ \\
\hline Pork \& Beans & Breaded Chicken Sandwich & 6 & $51.1 \pm 9$ & $34.9 \pm 11.4$ & $43 \pm 7.6$ \\
\hline Mixed Normandy Vegetables & Hot Dog on a Bun & 6 & $71.5 \pm 17.6$ & $14.9 \pm 10$ & $43.2 \pm 11.1$ \\
\hline Veggie Dippers & Mini Chef Salad & 5 & $70.7 \pm 14.9$ & $18.2 \pm 24$ & $44.4 \pm 14.1$ \\
\hline Veggie Dippers & Cheese or Sausage Pizza & 5 & $66.8 \pm 10.9$ & $22.7 \pm 9.9$ & $44.8 \pm 6.2$ \\
\hline
\end{tabular}




\section{Continued}

\begin{tabular}{|c|c|c|c|c|c|}
\hline Sonoma Vegetables & Chef Boyardee Ravioli & 5 & $82.7 \pm 10.2$ & $7.6 \pm 3.3$ & $45.2 \pm 5.6$ \\
\hline Potato Wedges & Grilled Chicken Sandwich & 6 & $49.2 \pm 18.9$ & $41.9 \pm 9.1$ & $45.6 \pm 12.5$ \\
\hline Whole Dill Pickle & Deli Sandwich & 6 & $45.4 \pm 18.6$ & $46.4 \pm 16.9$ & $45.9 \pm 16.5$ \\
\hline French Fries & Pizza Sandwich & 5 & $49.8 \pm 14.2$ & $42.1 \pm 14.2$ & $46 \pm 11.7$ \\
\hline Mashed Potatoes w Gravy & Mini Sub Sandwich & 5 & $55.7 \pm 24.9$ & $39.2 \pm 28.5$ & $47.5 \pm 26.4$ \\
\hline Whole Kernel Corn & Cheese or Sausage Pizza & 6 & $69.4 \pm 11.6$ & $26.4 \pm 12.6$ & $47.9 \pm 11.4$ \\
\hline Mixed Normandy Vegetables & Sea Shaped Fish Sticks & 10 & $75.3 \pm 10.9$ & $20.6 \pm 12.9$ & $48 \pm 8.8$ \\
\hline Steamed Broccoli & Chicken Tender Snack Wrap & 1 & $67.7 \pm 0$ & $28.8 \pm 0$ & $48.2 \pm 0.0$ \\
\hline Raw Baby Carrots and Celery & Deli Wrap & 3 & $62.6 \pm 11$ & $34.3 \pm 19.7$ & $48.4 \pm 5.5$ \\
\hline Green Peas & Cheese or Pepperoni Pizza & 9 & $71.5 \pm 19.3$ & $26.1 \pm 10.7$ & $48.8 \pm 10.0$ \\
\hline Veggie Dippers & Sunbutter Sandwich & 3 & $43 \pm 8.9$ & $55.6 \pm 26$ & $49.3 \pm 14.1$ \\
\hline Corn on the Cob & Chicken Tender Snack Wrap & 6 & $70.9 \pm 12.7$ & $28.2 \pm 10.8$ & $49.6 \pm 11.0$ \\
\hline Veggie Dippers & Chef Boyardee Ravioli & 3 & $73.9 \pm 13.6$ & $26 \pm 21.6$ & $49.9 \pm 6.2$ \\
\hline Sonoma Vegetables & Chicken Spaghetti & 2 & $65.6 \pm 31.5$ & $35.8 \pm 12.5$ & $50.7 \pm 22.0$ \\
\hline Whole Kernel Corn & Cheese or Pepperoni Pizza & 6 & $74.8 \pm 14$ & $27.2 \pm 5.3$ & $51 \pm 5.9$ \\
\hline Sweet Potato Fries & Hamburger or Cheeseburger & 1 & $91.4 \pm 0$ & $11.1 \pm 0$ & $51.2 \pm 0.0$ \\
\hline Raw Sweet Potato Sticks & Chicken Fried Steak Sandwich & 4 & $66.1 \pm 20.2$ & $42.4 \pm 17.5$ & $54.2 \pm 16.8$ \\
\hline Mashed Potatoes w Gravy & Deli Sandwich & 6 & $63.9 \pm 17.7$ & $46.4 \pm 16.9$ & $55.1 \pm 14.6$ \\
\hline Baked Beans & Chicken Fried Steak Sandwich & 6 & $63.4 \pm 13.4$ & $47.1 \pm 17.1$ & $55.3 \pm 6.1$ \\
\hline Green Beans & Mini sub Sandwich & 4 & $70.1 \pm 17.7$ & $43.4 \pm 31.1$ & $56.7 \pm 13.3$ \\
\hline Sweet Potato Fries & BBQ on a Bun & 2 & $88.2 \pm 5.1$ & $32.4 \pm 12.5$ & $60.3 \pm 8.8$ \\
\hline Veggie Dippers & Cheesy Baked Potato & 4 & $59.8 \pm 24.1$ & $63.6 \pm 24$ & $61.7 \pm 24.0$ \\
\hline Whole Kernel Corn & Cheesy Baked Potato & 4 & $72.7 \pm 23.2$ & $54.4 \pm 16$ & $63.5 \pm 12.1$ \\
\hline Steamed Broccoli w/cheese & Deli Wrap & 1 & $86.8 \pm 0$ & $42.3 \pm 0$ & $64.5 \pm 0.0$ \\
\hline Raw Sweet Potato Sticks & Baked Chicken on Bone & 4 & $47.7 \pm 23.3$ & $85.7 \pm 5.7$ & $66.7 \pm 14.3$ \\
\hline Baked Beans & Baked Chicken on Bone & 6 & $69.8 \pm 16.2$ & $84.5 \pm 4.9$ & $77.1 \pm 8.8$ \\
\hline Green Peas & Sunbutter Sandwich & 1 & $89.1 \pm 0$ & $69.3 \pm 0$ & $79.2 \pm 0.0$ \\
\hline All & All & 305 & $57.7 \pm 21.2$ & $29.3 \pm 20.5$ & $43.5 \pm 14.9$ \\
\hline
\end{tabular}

${ }^{\mathrm{a}}$ Values are mean $\% \pm$ standard deviations; ${ }^{\mathrm{b}}$ Ranked from smallest to largest total percent wasted; ${ }^{\mathrm{c}}$ Here $n$ in the number of observations for each entrée/vegetable pairing. 
vegetable side dish resulted in a notable amount of plate waste for both entrée (44.3\%) and vegetable (80.8\%). Although chicken nuggets had the lowest percent plate waste when served with green beans, the percentage plate waste for green beans was among the highest (69\%) and was higher than the plate waste for chicken nuggets and mashed potatoes and gravy.

Four out of five pairings that had the lowest overall plate waste involved white potato products. Entrées and vegetables pairings with the least overall plate waste involved the most popular entrées and the most popular vegetables for elementary school students.

Five most wasted pairings of entrée and vegetables were corn on the cob (80.8\%) served with deli sliders (44.3\%), hamburger or cheeseburger (37.4) with ranch style beans (78.5\%), pizza (27.1\%) with corn on the cob (81.3\%), pizza (28.8\%) with green peas (78.1\%) and barbeque on a bun (39.1\%) with corn on the cob (63.0\%).

Similar to our findings for the pre-implementation period of nutrition standards, four out of five entrée and vegetable pairings that had the lowest overall plate waste involved potato products (tater tots, mashed potato with gravy, French fries) as side dishes for the period post-implementation of the new school meal standards (Table 5). Broccoli florets served with a hot dog on a bun were among the five least wasted entrée and vegetable pairings.

Post-implementation of the new standards, the mean combined percent plate waste for entrée and vegetable pairings increased slightly from $40.4 \%$ to $43.5 \%$ but this increase was not statistically significant (Table 4 and Table 5). Mean percent plate waste for vegetables, both pre- and post-implementation of the new school meal standards, were over 50\% (52.1\% for pre-implementation and 57.7\% for post-implementation). Entrées were wasted less compared to vegetables. Mean entrée plate waste increased by less than 1\% post- compared to pre-implementation (28.6\% for pre- and $29.3 \%$ for post-implementation).

\subsection{Energy and Nutrients Available and Consumed from School Meals}

The results reported in Table 4 and Table 5 raise a set of questions regarding the energy and nutrients intake from the most and the least wasted entrées and vegetables and their pairings. The nutrient profile for every entrée and vegetable sampled was provided by school district. The mean energy and nutrients intakes per student from entrée and vegetable pairing were calculated using total energy and nutrients provided per serving and the percentage of plate waste. Table 6 and Table 7 report mean energy and nutrients (dietary fiber, sodium, iron, vitamin A, vitamin C, calcium, protein, fat, and saturated fat) intake per student and mean energy and nutrients available to students from entrée/vegetable pairings from school meals for the ten most and least wasted entrée and vegetable pairings, pre- and post-implementation of the new standards, respectively ${ }^{2}$. For the ten most wasted pairings, the mean combined waste from entrée/vegetable pairings was 51\% pre-implementation and $60 \%$ post-implementation versus for the ten least wasted pairings, the mean combined waste from entrée/vegetable pairings was $31 \%$ both pre-implementation and post-implementation of the new school meal standards.

With respect to the ten least wasted pairings, more calories were offered post-implementation (377 kcal/student) compared to pre-implementation (247 kcal/student) and consequently more calories were consumed post- (260 kcal/student) compared to pre-implementation (240 kcal/student). Less sodium was offered post- (800 mg/student) compared to pre-implementation (889 mg/student). No notable differences in the amount of saturated fat offered were observed between pre- and post-implementation. In general, more dietary fiber, iron, vitamin A and C, calcium and protein were available to students from entrée/vegetable pairings served post-implementation compared to pre-implementation of the new school meal standards. Specifically, more dietary fiber were available to students during the post- compared to pre-implementation (5 g/student versus 3.2 g/student) and noticeably more vitamin C was available to students from food pairings post- compared to preimplementation (16.5 mg/student versus $7.3 \mathrm{mg} / \mathrm{student}$ ).

Similar patterns of nutrients available to students from the entrée/vegetable pairings were observed for the ten most wasted food pairings. Noticeable increases in the amounts of vitamin A and C as well as calcium available to students were observed for the post- compared to pre-implementation period.

After the changes in school meal standards, the minimum and maximum energy required for lunch for kindergarten through fifth grade are between 550 - 650 kcal. For our sample of the ten least and most wasted pairings, mean energy available from entrée/vegetable pairings was 365 kcal/student pre-implementation and 382

\footnotetext{
${ }^{2}$ In this paper, we reported only the results for the ten most and least wasted entrée/vegetables pairing due to space limitations. A full summary of results is available from the authors upon request.
} 
Table 6. Mean intakes of energy and nutrients mean energy and nutrientsavailable from combined entrée/vegetable pairings, per student, pre-implementation of the new school meal standards.

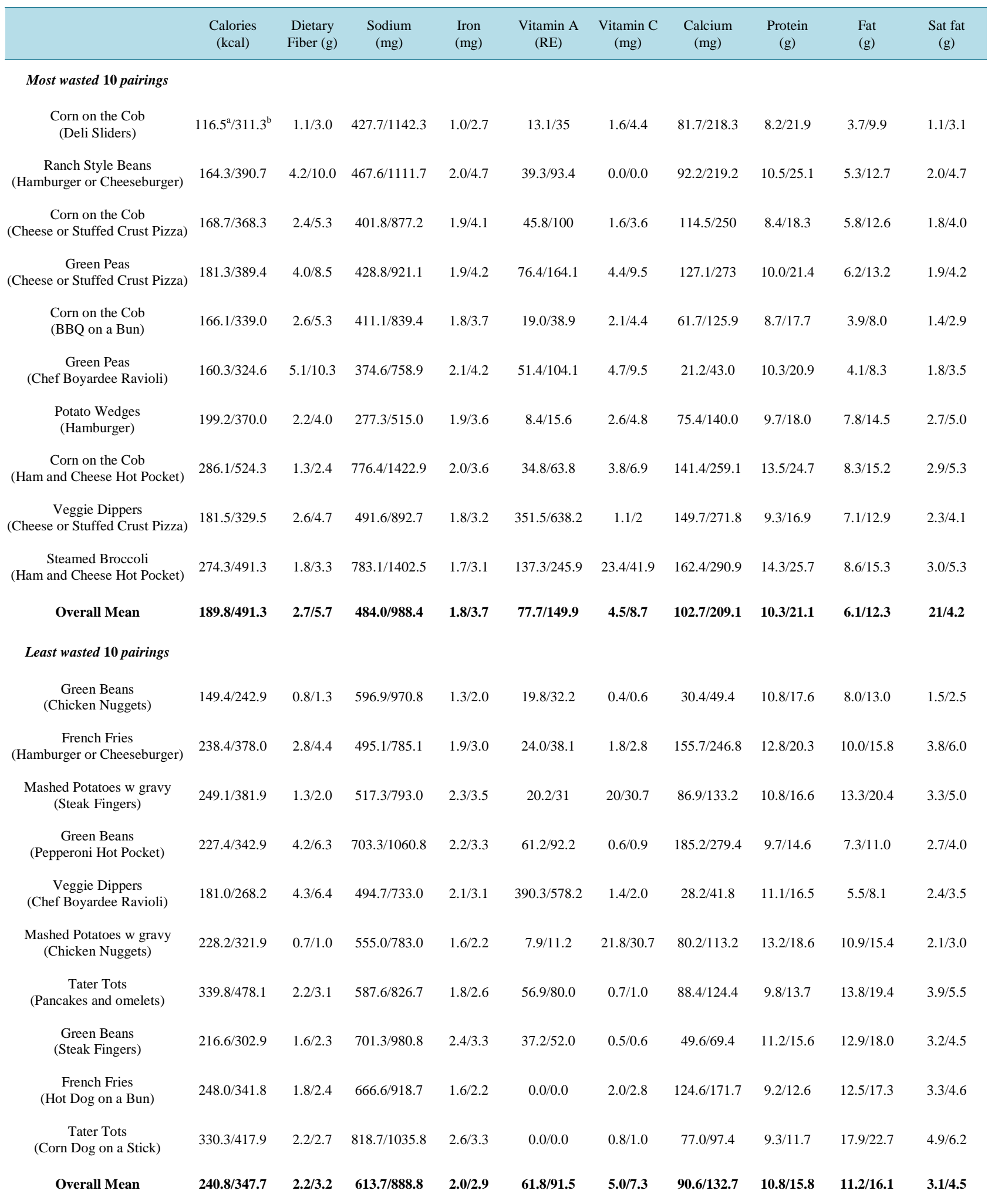

${ }^{\mathrm{a}}$ Mean energy or nutrient intake from the combined entrée and vegetable pairing; ${ }^{\mathrm{b}}$ Mean energy or nutrient available from the combined entrée and vegetable pairing. 
Table 7. Mean intakes of energy and nutrients and mean energy and nutrients available from combined entrée/vegetable pairings, per student, post-implementation of the new school meal standards.

\begin{tabular}{|c|c|c|c|c|c|c|c|c|c|c|}
\hline & $\begin{array}{l}\text { Calories } \\
\text { (kcal) }\end{array}$ & $\begin{array}{l}\text { Dietary } \\
\text { Fiber(g) }\end{array}$ & $\begin{array}{l}\text { Sodium } \\
\text { (mg) }\end{array}$ & $\begin{array}{l}\text { Iron } \\
(\mathrm{mg})\end{array}$ & $\begin{array}{l}\text { Vitamin A } \\
\quad(\mathrm{RE})\end{array}$ & $\begin{array}{l}\text { Vitamin C } \\
(\mathrm{mg})\end{array}$ & $\begin{array}{l}\text { Calcium } \\
\text { (mg) }\end{array}$ & $\begin{array}{l}\text { Protein } \\
\text { (g) }\end{array}$ & $\begin{array}{l}\text { Fat } \\
(\mathrm{g})\end{array}$ & $\begin{array}{l}\text { Satfat } \\
\text { (g) }\end{array}$ \\
\hline \multicolumn{11}{|l|}{ Most wasted 10 pairings } \\
\hline $\begin{array}{c}\text { Green Peas } \\
\text { (Sunbutter Sandwich) }\end{array}$ & $77.8^{\mathrm{a}} / 374.1^{\mathrm{b}}$ & 2.8/13.3 & 87.0/418.6 & 0.8/3.6 & 13.3/64.1 & $2.0 / 9.5$ & 25.5/122.9 & 4.1/19.9 & $3.8 / 18.2$ & $0.4 / 2.0$ \\
\hline $\begin{array}{c}\text { Steamed Broccoli w/cheese Sauce } \\
\text { (Deli Wrap) }\end{array}$ & $138.5 / 390.3$ & $1.6 / 4.5$ & $630.7 / 1777.3$ & $0.8 / 2.2$ & 255.8/720.9 & $15.2 / 42.9$ & 183.9/518.2 & $7.9 / 22.4$ & $6.6 / 18.7$ & $2.1 / 5.8$ \\
\hline $\begin{array}{l}\text { Whole Kernel Corn } \\
\text { (Cheesy Baked Potato) }\end{array}$ & $128.7 / 352.8$ & 2.6/7.1 & 177.2/485.6 & $0.8 / 2.2$ & 39.9/109.5 & $17.8 / 48.7$ & 121.9/334.1 & $6.4 / 17.5$ & $2.5 / 6.8$ & $1.4 / 3.9$ \\
\hline $\begin{array}{c}\text { Veggie Dippers } \\
\text { (Cheesy Baked Potato) }\end{array}$ & $116.3 / 303.5$ & $2.5 / 6.5$ & 202.2/527.7 & $0.8 / 2.2$ & 241.1/629.3 & $18.3 / 47.8$ & $135.4 / 353.4$ & $6.1 / 15.8$ & $2.4 / 6.4$ & $1.5 / 3.9$ \\
\hline $\begin{array}{l}\text { Sweet Potato Fries } \\
\text { (BBQ on a Bun) }\end{array}$ & $182.4 / 459.0$ & $2.5 / 6.3$ & 368.8/928.4 & $1.2 / 3.0$ & $0.0 / 0.0$ & $1.7 / 4.4$ & $58.0 / 145.9$ & $7.0 / 17.7$ & $6.3 / 16.0$ & $1.4 / 3.4$ \\
\hline $\begin{array}{c}\text { Green Beans } \\
\text { (Mini sub Sandwich) }\end{array}$ & $114.3 / 264.2$ & $1.0 / 2.3$ & $693.0 / 1602.0$ & $1.0 / 2.2$ & 29.1/67.2 & $0.6 / 1.4$ & $107.2 / 247.7$ & $8.9 / 20.5$ & $4.3 / 9.9$ & $1.3 / 3.1$ \\
\hline $\begin{array}{c}\text { Baked Beans } \\
\text { (Chicken Fried Steak Sandwich) }\end{array}$ & $246.4 / 550.9$ & 4.3/9.6 & $770 / 1721.2$ & $2.0 / 4.6$ & $17.8 / 39.8$ & $0.7 / 1.5$ & 70.6/157.8 & $12.9 / 28.7$ & $7.7 / 17.2$ & $2.3 / 5.0$ \\
\hline $\begin{array}{l}\text { Mashed Potatoes w gravy } \\
\text { (Deli Sandwich) }\end{array}$ & $95.1 / 211.9$ & 1.3/3.0 & $225.7 / 503.0$ & $0.8 / 1.9$ & 5.0/11.2 & $13.8 / 30.7$ & 46.3/103.2 & $2.5 / 5.6$ & $1.8 / 3.9$ & $0.5 / 1.0$ \\
\hline $\begin{array}{c}\text { Raw Sweet Potato Sticks } \\
\text { (Chicken Fried Steak Sandwich) }\end{array}$ & $230.4 / 503.5$ & 3.6/8.0 & 527.4/1152.6 & $2.3 / 4.9$ & $1220.3 / 2667$ & $2.0 / 4.4$ & 82.2/179.6 & $11 / 24.1$ & 7.1/15.6 & $2.1 / 4.5$ \\
\hline $\begin{array}{c}\text { Sweet Potato Fries } \\
\text { (Hamburger or Cheeseburger) }\end{array}$ & $227.0 / 465.4$ & $2.9 / 6.0$ & $378.9 / 776.9$ & $1.6 / 3.2$ & $0.0 / 0.0$ & $1.8 / 3.6$ & 117.6/241.3 & $10.5 / 21.5$ & $10.0 / 20.5$ & $2.8 / 5.8$ \\
\hline Overall Mean & $155.7 / 387.6$ & 2.5/6.7 & 406.1/989.3 & $1.2 / 3.0$ & $182.2 / 430.9$ & $7.4 / 19.5$ & $94.9 / 240.4$ & 7.7/19.4 & $5.3 / 13.3$ & $1.6 / 3.8$ \\
\hline \multicolumn{11}{|l|}{ Least wasted 10 pairings } \\
\hline $\begin{array}{c}\text { Garden Salad w/ranch } \\
\text { (Cheese Stuffed Bread Stick) }\end{array}$ & $233.1 / 353.9$ & $3.1 / 4.6$ & 649.2/985.6 & $1.7 / 2.7$ & $146.8 / 222.8$ & $3.8 / 5.8$ & $245.3 / 372.4$ & $10.0 / 15.2$ & $10.4 / 15.7$ & $3.0 / 4.6$ \\
\hline $\begin{array}{l}\text { Mashed Potatoes w gravy } \\
\text { (Popcorn Chicken) }\end{array}$ & $251.0 / 380.5$ & $1.5 / 2.3$ & $398.5 / 604.1$ & $1.7 / 2.6$ & 23.9/36.3 & $20.3 / 30.7$ & 78.0/118.3 & 11.0/16.7 & $13.2 / 20.0$ & $2.4 / 3.7$ \\
\hline $\begin{array}{c}\text { Potato Wedges } \\
\text { (Hamburger or Cheeseburger) }\end{array}$ & $270.0 / 405.4$ & $2.7 / 4.0$ & $447.5 / 671.9$ & $2.4 / 3.6$ & $30.6 / 46.0$ & $3.2 / 4.8$ & $160.7 / 241.3$ & $14.3 / 21.5$ & 11.0/16.5 & $4.2 / 6.3$ \\
\hline $\begin{array}{c}\text { Garden Salad w/ranch } \\
\text { (Italian Spaghetti \& Meat Sauce) }\end{array}$ & $275.8 / 413.9$ & $5.5 / 8.3$ & $369.6 / 554.7$ & $2.9 / 4.3$ & $242 / 363.1$ & $13.5 / 20.3$ & 149.0/223.6 & $16.4 / 24.6$ & $9.5 / 14.2$ & $2.9 / 4.4$ \\
\hline $\begin{array}{c}\text { French Fries } \\
\text { (Hamburger or Cheeseburger) }\end{array}$ & $267.2 / 387.2$ & $3.0 / 4.4$ & $569.7 / 825.7$ & $2.0 / 3.0$ & $31.7 / 46.0$ & $1.9 / 2.8$ & $188.4 / 273.0$ & $14.6 / 21.2$ & $11.2 / 16.3$ & $4.4 / 6.4$ \\
\hline $\begin{array}{l}\text { Broccoli florets } \\
\text { (Hot Dog on a Bun) }\end{array}$ & $189.5 / 265.5$ & $2.1 / 3.0$ & $552.9 / 774.7$ & $1.9 / 2.7$ & $82.4 / 115.5$ & $47.7 / 66.9$ & $110.8 / 155.2$ & $9.4 / 13.1$ & 9.8/13.8 & $2.5 / 3.5$ \\
\hline $\begin{array}{l}\text { Mashed Potatoes w gravy } \\
\text { (Chicken Nuggets) }\end{array}$ & $230.2 / 321.9$ & $0.7 / 1.0$ & $559.8 / 783.0$ & $1.6 / 2.2$ & 8.0/11.2 & $22 / 30.7$ & $80.9 / 113.2$ & 13.3/18.6 & $11.0 / 15.4$ & $2.2 / 3.0$ \\
\hline $\begin{array}{c}\text { Tater Tots } \\
\text { (Beef \& Bean Burrito) }\end{array}$ & $262.5 / 360.9$ & $5.5 / 7.6$ & 438.9/603.5 & $2.4 / 3.4$ & $1.5 / 2.1$ & $1.6 / 2.1$ & $51.9 / 71.3$ & 10.0/13.7 & $10.8 / 14.9$ & $2.8 / 3.9$ \\
\hline $\begin{array}{c}\text { Tater Tots } \\
\text { (Hamburger or Cheeseburger) }\end{array}$ & $319.3 / 433.3$ & $3.5 / 4.7$ & 702.1/952.7 & $2.6 / 3.5$ & $33.9 / 46.0$ & $0.8 / 1.0$ & 175.9/238.7 & $15.7 / 21.3$ & $15.6 / 21.2$ & 5.5/7.4 \\
\hline Overall Mean & 259.3/377.3 & $3.4 / 5.0$ & $550.7 / 800.6$ & $2.2 / 3.3$ & 64.0/94.9 & 11.5/16.5 & 135.9/198.7 & 13.0/19.0 & 11.3/16.5 & $3.2 / 4.6$ \\
\hline
\end{tabular}

${ }^{\mathrm{a}}$ Mean energy or nutrient intake from the combined entrée and vegetable pairing; ${ }^{\mathrm{b}}$ Mean energy or nutrient available from the combined entrée and vegetable pairing. 
$\mathrm{kcal} / \mathrm{student}$ post-implementation. The target requirement for sodium for lunch is $\leq 1230 \mathrm{mg}$ that started in SY 2014-2015. For our sample, the mean sodium available from entrée/vegetable pairings from school meals were $938 \mathrm{mg} / \mathrm{student}$ pre- and $895 \mathrm{mg} / \mathrm{student}$ post-implementation. The new standards require that saturated fat should be less than $10 \%$ total calories. Saturated fat available to students from entrée/vegetable pairings in our sample was less than $1 \%$ of total calories available to students in both the pre- and post-implementation periods of the new school meal standards.

\section{Discussion}

Existing behavioral and experimental studies that looked at the relationship between main dish and side dishes found that about $50 \%$ of the variability in overall meal acceptance ratings could be explained by liking/disliking of the main entrée, $16 \%$ and $12 \%$ could be explained by starches and the vegetables, respectively, and $7 \%$ by the salad [17]. The same entrée presented with different side dishes can get different liking ratings compared to rating only the entrée by itself [18]. Jimenez et al. found that when the main entrée was served with well-liked side dishes, the main entrée was liked less. In contrast, if side dishes are disliked by individuals, the main dish will receive higher liking scores [18]. Our findings from elementary school lunches, therefore, are consistent with those from previous behavioral and experimental studies. We observed that specific pairings of entrées and vegetables reduced total food waste. Pairings of more popular entrées with less popular vegetables resulted in higher vegetable waste. Specifically, chicken nuggets were wasted less when paired with green beans and wasted more when paired with mashed potatoes. Compared to pairing of green beans with chicken nuggets, green beans were wasted less when paired with steak fingers and pepperoni hot pockets. In general, pairing of popular entrées, such as chicken nuggets and burgers, with popular vegetables, such as potato products, resulted in lower combined plate waste.

In order to meet the new nutrition standards, schools in our sample offered more selection of vegetables and modified recipes post-implementation of the new standards, but the serving sizes did not change. Overall, our results indicated that more nutritious meals were offered during the post-implementation period compared to the pre-implementation period. The new school meal standards had no effect on the entrée plate waste and led to a small insignificant increase in the combined plate waste from entrée and vegetable pairings (40.4\% pre- and 43.5\% post-implementation). Modification of the recipes and possibly less familiarity with some of the vegetables offered may have contributed to the increased vegetable waste observed post-implementation. Further research is needed to understand this dynamic and if food pairings can reduce food waste and cost. Other strategies to reduce plate waste include adjusting portion sizes to child age and involving students in taste-testing of new food offerings.

Relatively few studies examined plate waste after the implementation of the new standards. Byker and et al. (2014) collected food waste data from one pre-kindergarten and five kindergarten classes from one elementary school for one full week in March 2013, after the new standards went into effect. They found that $45.3 \%$ of all food that was served was wasted with vegetables being wasted the most (51.4\%) followed by the main entrée (51.0\%). Cohen et al. (2014) measured plate waste from four schools (grades 3-8), with two data collection days per school, in an urban low-income district, in which roughly $85 \%$ of the students were eligible to receive free or reduced-price meals. The analysis was conducted using the data from before and after the implementation of new standards. They found that, although students' consumption of entrées and vegetables increased after the implementation of the new standards, roughly $24.9 \%$ of vegetables and $72.3 \%$ of entrées were consumed before the implementation of the new standards. About $41.1 \%$ of vegetables and $87.9 \%$ of entrées were consumed after the implementation. Schwartz et al. (2015) collected data from 12 middle schools (5th to 7th grade) located in an urban, low-income district with over $70 \%$ of students qualifying for free lunches and $13 \%$ qualifying for reduced-price lunches. They found that the percentage of vegetable servings consumed increased from $45 \%$ to $64 \%$ before versus after the changes in standards. The consumption of entrées also increased from $71 \%$ to $84 \%$ before versus after the changes in standards. Their study concluded that the revised NSLP nutrition standards and policies led to more nutritious meals and less overall plate waste.

This current study has several limitations. First, plate waste information was collected for main entrées and vegetables only. We did not collect data on fruit selection and waste of fruit, milk or grains. Consumption of these meal components could have affected plate waste of the entrées and vegetables. Second, the data for preand post-implementation of the new standards were collected at different times of the school year, spring seme- 
ster for pre-implementation and fall semester for post-implementation. It is possible that participation in school meals and potential plate waste may have differed not only because of the changes in standards but also due to the time of the school year. Third, students' entrée and vegetable choices in our study were predetermined by menus designed by school food service administrators. Students had limited choices as to what vegetables were available with a particular entrée. In order to fully understand the actual effect of entrée/vegetable pairings on plate waste, each potential entrée would need to be paired with each potential vegetable and there would need to be sufficient power to detect significant differences. This situation would require greater control over the schools' menu than is possible in a natural setting. Even so, our observations suggest that school food service administrators may reduce plate waste from school meals as well as incur fewer wasted dollars for foodservice by optimizing entrée/vegetable pairings. On an average school day, school lunch participants consume more vegetables at school compared to nonparticipants [19]. As a result of serving tasty and nutritious meals, students may enjoy and waste less of their school meals, which is especially important for those students relying on school meals for most of their energy and nutrient needs.

\section{Conclusion}

Pairings of entrées and vegetables are an important consideration when assessing plate waste among elementary school children, and lost dollars to school food service. A relationship was noted between entrées and vegetables suggesting that greater consumption of certain entrées was associated with greater waste of certain vegetables. It was observed that popular entrées, such as burgers and chicken nuggets, contributed to greater waste of less popular vegetables, such as green beans and sweet potato fries. However, it was unclear if decreased popularity of a vegetable was a direct result of the vegetable itself, or of the child having "filled up" on a popular paired entrée, indirectly causing decreased consumption of the vegetable. Entrées paired with popular vegetables such as potatoes (served as tater tots, oven-baked French fries, and wedges) experienced the least amount of waste. In view of these findings, understanding entrée/vegetable pairings may assist schools in serving tasty yet nutritious meals while maintaining high program participation rates and staying within constrained operating budgets.

\section{Acknowledgements}

The authors acknowledge the Alliance for Potato Research and Education for providing funding for this research. The authors would like to thank Lindsey Field, research assistants and school food service directors and personnel for their assistance in data collection. All authors have read and approved the final manuscript.

\section{References}

[1] IOM (Institute of Medicine) (2009) School Meals: Building Blocks for Healthy Children. The National Academies Press, Washington DC.

[2] U.S. Department of Agriculture and U.S. Department of Health and Human Services (2010) Dietary Guidelines for Americans 2010. Washington DC.

[3] White House Task Force on Childhood Obesity Report to the President. Solving the Problem of Childhood Obesity within a Generation. http://www.letsmove.gov/white-house-task-force-childhood-obesity-report-president.

[4] U.S. Department of Agriculture (2012) Nutrition Standards in the National School Lunch and School Breakfast Programs; final rule. Federal Register, 4088-167.

[5] Buzby, J.C. and Guthrie, J.F. (2002) Plate Waste in School Nutrition Programs: Final Report to Congress. Economic Research Service, U.S. Department of Agriculture, Washington DC.

[6] GAO (2014) Implementing Nutrition Changes Was Challenging and Clarification of Oversight Requirements is Needed. U.S. Government Accountability Office Report, Washington DC.

[7] Byker, C.J., Farris, A.R., Marcenelle, M., Davis, G.C. and Serrano, E.L. (2014) Food Waste in a School Nutrition Program after Implementation of New Lunch Program Guidelines. Journal of Nutrition Education and Behavior, 46, 406411. http://dx.doi.org/10.1016/j.jneb.2014.03.009

[8] Cohen, J.F., Richardson, S., Austin, S.B., Economos, C.D. and Rimm, E.B. (2013) School Lunch Waste among Middle School Students: Nutrients Consumed and Costs. American Journal of Preventive Medicine, 44, 114-121. http://dx.doi.org/10.1016/j.amepre.2012.09.060

[9] Schwartz, M.B., Henderson, K.E., Read, M., Danna, N. and Ickovics, J.R. (2015) New School Meal Regulations Increase Fruit Consumption and Do Not Increase Total Plate Waste. Childhood Obesity, 11, 242-247. 
[10] Gase, L.N., McCarthy, W.J., Robles, B. and Kuo, T. (2014) Student Receptivity to New School Meal Offerings: Assessing Fruit and Vegetable Waste among Middle School Students in the Los Angeles Unified School District. Preventative Medicine, 67, S28-S33. http://dx.doi.org/10.1016/j.ypmed.2014.04.013

[11] Adams, M.A., Pelletier, R.L., Zive, M.M. and Sallis, J.F. (2005) Salad Bars and Fruit and Vegetable Consumption in Elementary Schools: A Plate Waste Study. Journal of the American Dietetic Association, 105, 1789-1792. http://dx.doi.org/10.1016/j.jada.2005.08.013

[12] Turner, L. and Chaloupka, F.J. (2014) Perceived Reactions of Elementary School Students to Changes in School Lunches after Implementation of the United States Department of Agriculture's New Meals Standards: Minimal Backlash, but Rural and Socioeconomic Disparities Exist. Childhood Obesity, 10, 349-356.

[13] Cohen, J.F., Richardson, S., Parker, E., Catalano, P.J. and Rimm, E.B. (2014) Impact of the New U.S. Department of Agriculture School Meal Standards on Food Selection, Consumption, and Waste. American Journal of Preventive Medicine, 46, 388-394. http://dx.doi.org/10.1016/j.amepre.2013.11.013

[14] Smith, S.L. and Cunningham-Sabo, L. (2014) Food Choice, Plate Waste and Nutrient Intake of Elementary- and Middle-School Students Participating in the US National School Lunch Program. Public Health Nutrition, 17, 1255-1263. http://dx.doi.org/10.1017/S1368980013001894

[15] Bergman, E.A., Buergel, N.S., Englund, T.F. and Femrite, A. (2004) The Relationship of Meal and Recess Schedules to Plate Waste in Elementary Schools. Journal of Child Nutrition \& Management, 28.

[16] Institute of Education Sciences. Public Elementary/Secondary School Universe Survey, 2000-01, 2005-06, 2010-11, and 2011-12. https://nces.ed.gov/ccd/pubschuniv.asp

[17] Rogozenski, J.G. and Moskowitz, H.R. (1983) A System for the Preference Evaluation of Cyclic Menus. Foodservice Research International, 2, 139-161. http://dx.doi.org/10.1111/j.1745-4506.1983.tb00270.x

[18] Jimenez, M., Rodriguez, D., Greene, N., Zellner, D.A., Cardello, A.V. and Nestrud, M.(2015) Seeing a Meal Is Not Eating It: Hedonic Context Effects Differ for Visually Presented and Actually Eaten Foods. Food Quality and Preference, 41, 96-102. http://dx.doi.org/10.1016/j.foodqual.2014.11.015

[19] Ishdorj, A, Crepinsek, M.K. and Jensen, H.H. (2013) Children’s Consumption of Fruits and Vegetables: Do School Environment and Policies Affect Choices at School and Away from School? Applied Economic Perspectives and Policy, 35, 341-359. http://dx.doi.org/10.1093/aepp/ppt003 